\title{
Accuracy Analysis of Various Blood Pressure Measurement Methods by 119 Paramedics
}

\author{
Hyun-Jung Kim ${ }^{1}$, Jin-Hwa Kim², Jee-Hee Kim³ \\ ${ }^{1}$ Professor, Department of Nursing, Daewon University College, Jecheon-si, Chungbuk, 27135, Korea, ${ }^{2}$ Professor \\ Department of Emergency Medical Technology, Daewon University College, Jecheon-si, Chungbuk, 27135, Korea, \\ ${ }^{3}$ Professor Department of Emergency Medical Services, Kangwon National University, Samcheok-si, Gangwon-do, \\ 25949, Korea
}

\begin{abstract}
Background/Objectives: This study is to confirm the accuracy of various blood pressure measurement methods by 119 paramedics.

Methods/Statistical analysis: This study evaluated the accuracy of blood pressure measurement and the confidence of blood pressure measurement in stable, stable and noisy, driving, and driving noise situations for 40119 paramedics. The collected data were analysed with SPSS 22.0 using frequency, descriptive statistics, and paired t-test.

Findings: The confidence of blood pressure measurement was significantly higher in four situations after education. In blood pressure measurement accuracy, there was the significant difference in the following situations: Stable noisy and driving noisy in normal blood pressure $(\mathrm{t}=-2.823, p=.007)$. Stable driving, stable noisy $(\mathrm{t}=-2.191, p=.034)$, and driving noisy $(\mathrm{t}=-2.421, p=.020)$ in hypertension. Stable driving, stable noisy $(\mathrm{t}=-3.674, p=.001)$, and driving noisy $(\mathrm{t}=-2.095, p=.043)$ in hypotension. In the accuracy of blood pressure measurement by palpation, there was the significant difference in stable and driving situation under hypotension( $\mathrm{t}=-2.211, p=.033)$.
\end{abstract}

Improvements/Applications: The education on blood pressure measurement in various environments is necessary to improve the measurement accuracy of 119 emergency personnel.

Keywords: Blood Pressure, Paramedics, Measurement, Accuracy, Confidence

\section{Introduction}

The emergency medical system is largely divided into the pre-hospital stage and the hospital stage ${ }^{[1]}$. The pre-hospital stage includes patient rescue and

\section{Corresponding Author:}

\section{Jin-Hwa Kim}

Professor, Department of Emergency Medical

Technology, Daewon University College, Jecheon-si, Chungbuk, 27136, Korea

e-mail: choicekjh83@daewon.ac.kr

Phone Number: 82-10-9471-0635 transfer, patient evaluation and treatment, and severity classification, and the treatment of paramedics has the important influence on patient's prognosis ${ }^{[2]}$. In addition, the number of 119 paramedics who perform most of the first-aid and transfer tasks for patients at the pre-hospital stage is steadily increasing every year ${ }^{[3]}$.

The scope of work of first-class emergency medical personnel includes not only tasks such as CPR implementation in accordance with Article 33 of the Enforcement Rule of the Emergency Medical Act, but also the measurement of vital signs that are 
basically performed for evaluation and treatment of emergency patients. Vital sign is the most basic evaluation of a patient's clinical condition, an index that can predict the prognosis, and is an essential element for patient evaluation at the pre-hospital stage.In addition, the measurement of patient's vital sign at the pre-hospital stage is an essential factor in patient evaluation $^{[2]}$, and it is the fourth most frequent emergency treatment performed by 119 paramedics, and should be accurately measured because it is related to human life ${ }^{[4]}$. Among vital signs, blood pressure is a physiological variable that not only reflects the patient's hemodynamic factors, but also predicts the patient's current state and future state changes ${ }^{[5]}$.

Blood pressure measurement of emergency patients must be performed in the stable situation for high accuracy. But various, unpredictable and dangerous situations can occur at the site before the hospital. However, the field of paramedics at the prehospital stage varies from mild to severe patients, diseases and trauma, and dangerous situations that paramedics cannot predict may occur. In addition, blood pressure is often measured in unstable situations due to various disturbing factors such as people, vibration and noise. For this reason, it is very difficult to accurately measure blood pressure in various prehospital sites and ambulances where vibration and noise are generated ${ }^{[6]}$. Moreover, 119 paramedics are measuring blood pressure using auscultation, palpation, and automatic sphygmomanometer to determine the patient's condition ${ }^{[5]}$.. However, blood pressure that cannot be accurately measured has the risk of providing unnecessary or inappropriate treatment by erroneously judging the patient's condition, so accurate measurement of blood pressure is important for grasping the patient's condition ${ }^{[7,8]}$.

Most of the preceding studies related to blood pressure measurement are the thesis evaluating the knowledge and practice of blood pressure measurement for nurses and nursing students, or evaluating the knowledge and accuracy of blood pressure measurement of trainees and emergency rescue students ${ }^{[9-11]}$. In the results of previous studies involving 119 paramedics, palpation was the most preferred method of measuring blood pressure $(64.5 \%)$, and the accuracy of blood pressure measurement was measured by auscultation ${ }^{[5]}$.However, there has been no previous study confirming the accuracy of blood pressure measurement including the effect of noise in various situations considering the work characteristics of 119 paramedics.

Therefore, this study was attempted to provide the essential basic data for the development of blood pressure measurement guidelines and practical training programs to improve the quality of emergency medical services provided by 119 paramedics at all hospital sites by confirming the accuracy of various blood pressure measurement methods including the noise in the safe and driving situations of 119 paramedics.

\section{Method}

\subsection{Research design}

This study is the one-shot case experimental study attempted to confirm the accuracy of various blood pressure measurements for 119 paramedics.

\subsection{Research subjects and sampling method}

The subjects of this study were 119 paramedics from two fire departments located in J city and agreed to participate in the study. The number of study subjects was calculated as 34 when the significance level $(\alpha)$ was set to .05 , the power( $1-\beta)$ to .95 , and the effect size to .50 using $G^{*}$ Power 3.1.9. 40 people were selected in consideration of the dropout rate, and 40 people were included in total. 


\subsection{Research tools}

\subsubsection{The accuracy of blood pressure measurement} under stable conditions

Blood pressure in a stable situation was measured using the blood pressure measuring simulator BTCEAB2 (BT, Wonju) on a mat laid in the practice room under the assumption that the patient is lying down. As for the blood pressure measurement method, normal blood pressure, hypertension, and hypotension randomly designated by the researcher were measured by auscultation and palpation, and blood pressure measurement values were reported to the last digit. The auscultation method was utilized in brachial artery using the stethoscope while the palpation method being measured in the pulse of radial artery. The accuracy of blood pressure measurement accuracy in the stable situation means the difference between three blood pressure values set by the researcher and the subject's measurement value. The lower the score, the higher the blood pressure measurement accuracy.

2.3.2 The accuracy of blood pressure measurement in stable and noisy situations $(90 \mathrm{~dB})$

Blood pressure measurement in stable and noisy conditions was attempted at $90 \mathrm{~dB}$ in accordance with the $\ulcorner$ Rules on the Performance and Standards of Automobiles and Automobile Parts $\lrcorner$, and The method of blood pressure measurement using auscultation with palpation was tried in the same way as the accuracy of blood pressure measurement in the stable situation. The accuracy of blood pressure measurement accuracy in stable and noisy situations(90dB) refers to the difference between the three blood pressure values arbitrarily set by the researcher and the measured values obtained by the subject, and the lower the score, the higher the blood pressure measurement accuracy.

\subsubsection{The accuracy of blood pressure measurement} in driving situations
Medico-legal Update, January-March 2022, Vol.22, No. 133

The accuracy of blood pressure measurement in the driving situation means that it is calculated by traveling at the speed of $60 \mathrm{~km} / \mathrm{h}$ in $3 \mathrm{~km}$ section in accordance with the Road Traffic Act, and the blood pressure measurement method using auscultation with palpation was tried in the same way as the blood pressure measurement accuracy in the stable situation. The accuracy of blood pressure measurement in driving situations means the difference between the three blood pressure values set by the researcher and the subject's measurement values, and the lower the score, the higher the blood pressure measurement accuracy.

2.3.4 The accuracy of blood pressure measurement in driving and noisy situations $(90 \mathrm{~dB})$

Blood pressure measurement in a driving and noisy situation $(90 \mathrm{~dB})$ means calculating the blood pressure in $90 \mathrm{~dB}$ siren noise situation in addition to the driving situation at the speed of $60 \mathrm{~km} / \mathrm{h}$ over $3 \mathrm{~km}$ section. The blood pressure measurement method using auscultation with palpation was performed in the same way as the accuracy of blood pressure measurement in the stable situation. The accuracy of blood pressure measurement in driving with noisy conditions means the difference between three blood pressure values set by the researcher and the subject's measurement values, andthe lower the score, the higher the blood pressure measurement accuracy.

\subsubsection{The confidence of blood pressure} measurement

The blood pressure measurement confidence means each blood pressure measurement confidence using auscultation with palpation in stable and driving situations. It was measured on the NRS 0-10 Likert scale, and the higher the score, the higher the performance confidence. 


\subsection{Data collection}

The 119 paramedics working at the $\mathrm{J}$ city fire department for the study were given an explanation of the purpose, method, duration, expected effects, interests and possible problems of participation, and refusal to participate, including guaranteeing anonymity. Then, consent was submitted and the study was conducted. The study period was from July 22 to July 23, 2020.

\subsection{Data analysis}

The SPSS WINDOW 22.0 Program was used to analyze the collected data.The frequency and descriptive statistics were used to determine the general characteristics of the subject as well as the accuracy and confidence level of blood pressure measurement in four situations before and after education. The test for the difference between blood pressure measurement accuracy and confidence in four situations before and after training was analyzed by paired t-test.

\section{Results}

\subsection{General characteristics of subjects}

As for general characteristics of the subjects, the preferred method among gender, age, experience of 119 paramedics, clinical work experience, clinical work period, and blood pressure measurement method was investigated, and the analysis results are shown in Table 1. In terms of gender, there were $25(62.0 \%)$ women, the most common age was less than 25-30 years old, and the average age was 29.05 years. The most frequent experience of paramedics was less than 1-5 years(45.0\%), and the average experience was 32.55 months. 27(67.5\%) had clinical work experience, and 12(44.4\%) had a clinical work period of less than 1-3 years. The most common blood pressure measurement method was performed by palpation in the radial artery in 30 patients $(75.0 \%)$ (Table 1).

\subsection{Comparison of the accuracy and difference} in blood pressure measurement in four situations

The degree and the difference in the accuracy of blood pressure measurement in the four situations are shown in Table 2. There was no statistically significant difference in blood pressure measurement accuracy measured by auscultation between stable and driving conditions $(\mathrm{t}=-1695, \mathrm{p}=.098)$ at randomly set normal blood pressure. However, there was a statistically significant difference between stable noisy and driving noisy situation( $\mathrm{t}=-2.823, \mathrm{p}=.007)$. In hypertension, there was a statistically significant difference between stable and running situation $(\mathrm{t}=$ $2.191, \mathrm{p}=.034$ ), and between stable noisy and running noisy situations $(\mathrm{t}=-2.421, \mathrm{p}=.020)$. In hypotension, there was a statistically significant difference when comparing stable situation with driving one $(\mathrm{t}=-3.674$, $\mathrm{p}=.001$ ), stable noisy situation with driving noisy one $(\mathrm{t}=-2.095, \mathrm{p}=.043)$. In the accuracy of blood pressure measurement by palpation, there was a statistically significant difference between stable and driving situation $(\mathrm{t}=-2.211, \mathrm{p}=.033)($ Table 2$)$. 
Table 1. General Characteristics of Subjects $(\mathrm{N}=\mathbf{4 0})$

\begin{tabular}{|c|c|c|c|}
\hline Characteristics & Categories & $\mathbf{N}(\%)$ & Mean \pm SD \\
\hline \multirow{2}{*}{ Gender } & Male & $15(37.5)$ & \\
\hline & Female & $25(62.5)$ & \\
\hline \multirow{4}{*}{ Age } & $<20-25$ & $3(7.5)$ & \multirow{4}{*}{$\begin{array}{c}29.05 \pm 4.27 \\
\text { (months) }\end{array}$} \\
\hline & $<25-30$ & $24(60.0)$ & \\
\hline & $<30-35$ & $9(22.5)$ & \\
\hline & $\geq 35$ & $4(10.0)$ & \\
\hline \multirow{4}{*}{$\begin{array}{l}\text { Paramedic } \\
\text { career } \\
\text { (year) }\end{array}$} & $<1$ & $14(35.0)$ & \multirow{4}{*}{$\begin{array}{c}32.55 \pm 43.58 \\
\text { (months) }\end{array}$} \\
\hline & $<1-5$ & $18(45.0)$ & \\
\hline & $<5-10$ & $6(15.0)$ & \\
\hline & $\geq 10$ & $2(5.0)$ & \\
\hline \multirow{2}{*}{ Clinical work experie } & Yes & $27(67.5)$ & \\
\hline & No & $13(32.5)$ & \\
\hline \multirow{4}{*}{ Clinical work perio } & $<1$ & $1(3.7)$ & \\
\hline & $<1-3$ & $12(44.4)$ & \\
\hline & $<3-5$ & $9(33.3)$ & \\
\hline & $\geq 5$ & $5(18.5)$ & \\
\hline \multirow{3}{*}{$\begin{array}{l}\text { Blood pressure } \\
\text { Measurement } \\
\text { method }\end{array}$} & Stethoscope & $3(7.5)$ & \\
\hline & $\begin{array}{l}\text { Palpation } \\
\text { (radial) }\end{array}$ & $30(75.0)$ & \\
\hline & NIBP & $7(17.5)$ & \\
\hline
\end{tabular}


3.3. Comparison of the level and the difference of blood pressure measurement confidence in four situations before and after training

Table 3 shows the level and the difference of blood pressure measurement confidence in the four situations. In a stable situation, the confidence in blood pressure measurement through auscultation was 7.70 points before education and 8.18 points after education, but there was no statistically significant difference $(\mathrm{t}=-1.512, \mathrm{p}=.139)$. However, in the stable situation before and after education, there was a statistically significant difference in the confidence through palpation( $\mathrm{t}=-3.275, \mathrm{p}=.002)$, auscultation in stable noisy situation $(\mathrm{t}=-4.106, \mathrm{p}<.001)$, palpation in stable noisy situation $(\mathrm{t}=-5.414, \mathrm{p}<.001)$, auscultation in driving situation $(\mathrm{t}=-4.452, \mathrm{p}<.001)$, palpation in driving situation $(\mathrm{t}=-5.985, \mathrm{p}<.001)$, auscultation in driving noisy situation( $\mathrm{t}=-5.194, \mathrm{p}<.001)$, and palpation in driving noisy situation $(\mathrm{t}=-6.696,<.001)$ (table 3).

Table 2. Comparison of The Accuracy and The Difference of Blood Pressure Measurement in Four Situations $(\mathbf{N}=\mathbf{4 0})$

\begin{tabular}{|c|c|c|c|c|c|}
\hline \multicolumn{3}{|c|}{ Characteristics } & \multirow{2}{*}{$\begin{array}{c}\text { Mean } \pm \text { SD } \\
4.50 \pm 3.26\end{array}$} & \multirow{3}{*}{$\begin{array}{c}\mathbf{t} \\
-1.695\end{array}$} & \multirow{3}{*}{$\begin{array}{c}\mathbf{p} \\
.098\end{array}$} \\
\hline \multirow{12}{*}{ Auscultation } & \multirow{4}{*}{ Normal BP } & Stable & & & \\
\hline & & Driving & $6.81 \pm 7.63$ & & \\
\hline & & Stable+Noisy & $4.62 \pm 4.02$ & \multirow{2}{*}{-2.823} & \multirow{2}{*}{.007} \\
\hline & & Driving+Noisy & $8.12 \pm 6.71$ & & \\
\hline & \multirow{4}{*}{ Hypertension } & Stable & $6.37 \pm 7.16$ & \multirow{2}{*}{-2.191} & \multirow{2}{*}{.034} \\
\hline & & Driving & $11.06 \pm 10.58$ & & \\
\hline & & Stable+Noisy & $6.37 \pm 7.16$ & \multirow{2}{*}{-2.421} & \multirow{2}{*}{.020} \\
\hline & & Driving+Noisy & $9.81 \pm 7.19$ & & \\
\hline & \multirow{4}{*}{ Hypotension } & Stable & $3.12 \pm 2.45$ & \multirow{2}{*}{-3.674} & \multirow{2}{*}{.001} \\
\hline & & Driving & $6.87 \pm 5.53$ & & \\
\hline & & Stable+Noisy & $4.00 \pm 3.87$ & \multirow{2}{*}{-2.095} & \multirow{2}{*}{.043} \\
\hline & & Driving+Noisy & $6.32 \pm 7.01$ & & \\
\hline \multirow{12}{*}{ Palpation } & \multirow{4}{*}{ Normal BP } & Stable & $3.38 \pm 4.29$ & \multirow{2}{*}{-0.154} & \multirow{2}{*}{.878} \\
\hline & & Driving & $3.50 \pm 3.95$ & & \\
\hline & & Stable+Noisy & $2.63 \pm 4.08$ & 1620 & 111 \\
\hline & & Driving + Noisy & $3.50 \pm 5.57$ & -1.029 & .111 \\
\hline & & Stable & $3.38 \pm 4.58$ & 1246 & 220 \\
\hline & Hynertension & Driving & $6.00 \pm 9.28$ & -1.246 & .220 \\
\hline & пуретtension & Stable+Noisy & $3.50 \pm 4.41$ & & \\
\hline & & Driving+Noisy & $5.88 \pm 7.06$ & -0.706 & .484 \\
\hline & & Stable & $3.25 \pm 5.00$ & -2211 & \\
\hline & Hynotencion & Driving & $4.63 \pm 4.98$ & -2.211 & .033 \\
\hline & Нy putcision & Stable+Noisy & $2.88 \pm 3.18$ & 0508 & 553 \\
\hline & & Driving+Noisy & $3.38 \pm 5.35$ & -0.590 & .553 \\
\hline
\end{tabular}


Table 3. Comparison of The Level and The Difference of Blood Pressure Measurement Confidence in Four Situations $(\mathrm{N}=\mathbf{4 0})$

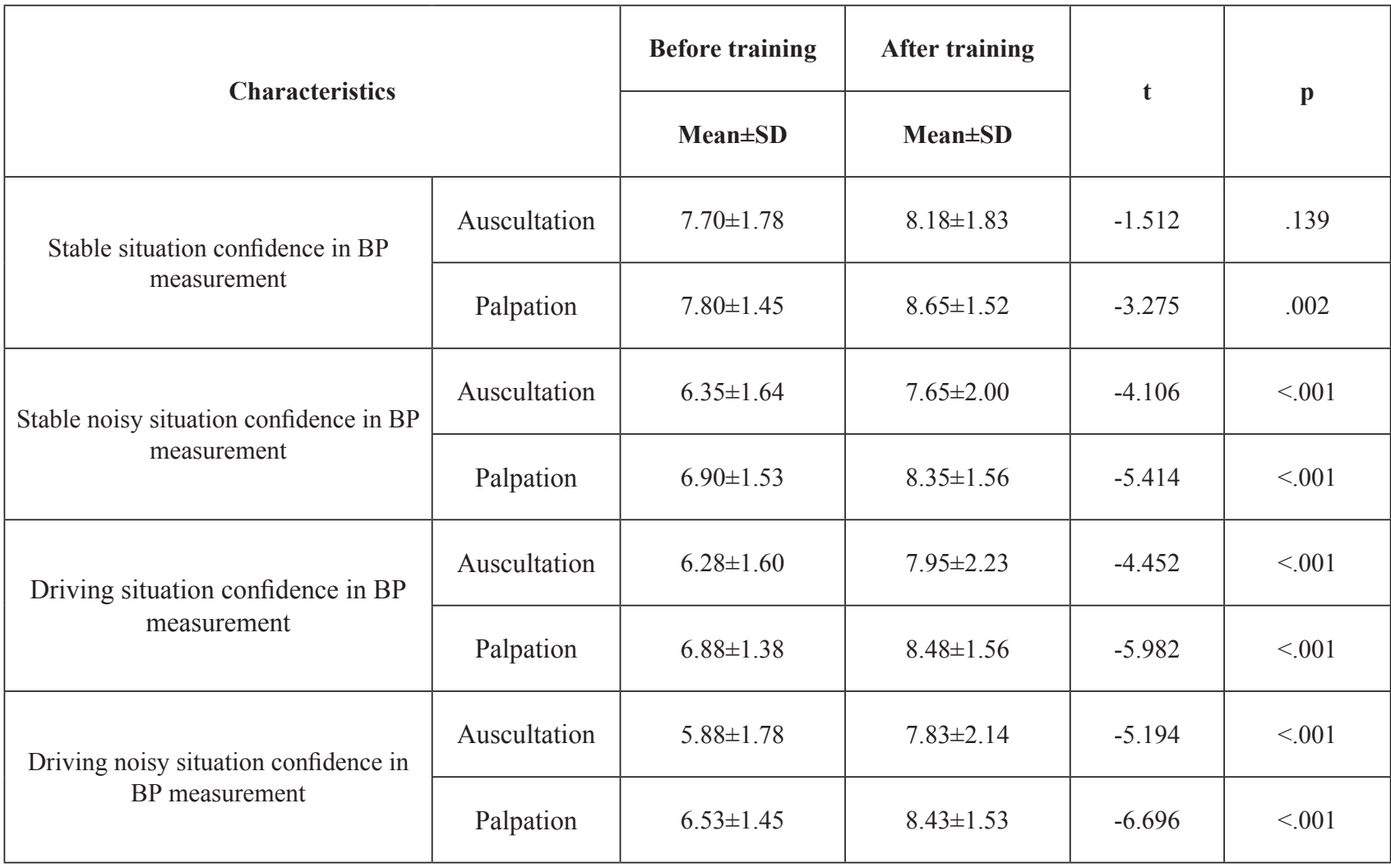

\section{Discussion}

In order to provide accurate first aid to patients at the pre-hospital stage, the patient's condition must be accurately determined. There are vital signs as a basic factor that can determine the patient's condition, among which blood pressure reflects the patient's basic condition. However, the emergency situations at pre-hospital stage are very diverse and unpredictable, and because there are many disturbing factors around the patients, it is difficult to accurately measure blood pressure, so education to measure blood pressure in various situations is required. Therefore, in this study, it was attempted to provide the basic data for educational program development by checking the accuracy of measurement in four situations of blood pressure measurement targeting 119 paramedics.

As a result of the study, there was no statistically significant difference between the accuracy of blood pressure measurement in stable conditions and the one during driving, measured with the stethoscope. However, the accuracy error range of blood pressure measurement in stable conditions was $4.50 \mathrm{mmHg}$ on average, and blood pressure measurement accuracy during driving was $6.81 \mathrm{mmHg}$, confirming that blood pressure measurement is more difficult in driving conditions than in stable conditions. This is the result supporting the previous study ${ }^{[5]}$ showing an error range of $4.79 \mathrm{mmHg}$ in stable conditions and $6.72 \mathrm{mmHg}$ in driving conditions in the blood pressure measurement accuracy for 119 paramedics. In addition, this result supports the result of a study ${ }^{[12]}$ that the accuracy of blood pressure measurement in an ambulance driving with severe vibration is more variable than in stable situation. In addition, there was a statistically significant difference in blood pressure measurement accuracy in stable noisy and driving 
noisy situations. And the accuracy error range of blood pressure measurement was the largest at $8.12 \mathrm{mmHg}$ in the driving noisy situation. In hypertension and hypotension, as with normal blood pressure, the one of blood pressure measurement was large in driving or noisy situation. This result was similar to the one of a study that compared the accuracy of systolic blood pressure measurement in normal blood pressure with the one in hypertensive conditions, and showed the large error range of blood pressure measurement accuracy in hypertension ${ }^{[13]}$. Therefore, it is necessary to provide various experience and education to the students of related departments to accurately measure the blood pressure, which is the basic data for grasping the patient's condition, and to develop the various practice training programs of blood pressure measurement that take into account the work characteristics of 119 paramedics.

As measured by palpation, under normal blood pressure, stable driving, stable noisy and driving noisy situation; under high blood pressure, stable noisy, stable noisy and driving noisy situation; in hypotension, stable noisy situation, driving noisy situation showed no significant difference in the accuracy of blood pressure measurement. This was different from the results of previous studies ${ }^{[5]}$ that confirmed the accuracy of blood pressure measurement in stable and driving situations. These results support the results of the study that 119 paramedics could measure most accurately because palpation was their most preferred method of measuring blood pressure $^{[11]}$. However, in order to accurately measure systolic and diastolic blood pressure, it is necessary to measure blood pressure using the stethoscope, so the experience of repeatedly measuring blood pressure with stethoscope in various situations is required. In addition, when evaluating the patient at the site prior to the hospital, based on the results of blood pressure measurement, the classification of patient's severity and rapid transfer are determined, and appropriate emergency managements are taken ${ }^{[11]}$. So the method of accurate blood pressure measurement should be emphasized.

In the results of the level of confidence in blood pressure measurement in four situations before and after education, in stable situation, the confidence in blood pressure determined by auscultation didn't show a significant difference before and after education. But in the stable situation, the confidence in blood pressure measurement by palpation was improved after education. This result is believed to be because the education of blood pressure measurement in stable situation is also repeated and generalized in universities and fire departments. The confidence in blood pressure measurement with auscultation or palpation methods in stable noisy, driving and driving noisy situations has improved statistically significantly after training compared to the one before training, which is believed to be the educational effect of how to measure blood pressure in new situations. Since the confidence in blood pressure measurement is correlated with the accuracy of blood pressure measurement ${ }^{[14]}$, the repeated education is necessary to improve the confidence in various situations.

\section{Conclusion}

This study attempted to confirm the accuracy of blood pressure measurements performed by 119 paramedics in various situations. As a result of the study, after training, the confidence of blood pressure measurement in various situations improved, and the accuracy of blood pressure measurement was lower in driving and noisy situations than in stable situations. These results are meaningful in verifying that the education of blood pressure measurement in various environments should be emphasized on 119 paramedics for the efficient clinical evaluation of patient and treatment in various field situations. Therefore, in addition to blood pressure measurement in the secure environment, the follow-up study can be 
recommended to confirm the effect after developing and applying the educational program for blood pressure measurement of 119 paramedics in various situations. Furthermore, it is suggested that this program should be reflected in practical education for the students in Department of Emergency Rescue.

Limitations: This study considered only paramedics intwo fire department and may not represent all paramedics.

Ethical Clearance:For ethical consideration of the study subjects, data was collected after approval through the deliberation(IRB No: D **-2020-01001-01) of Institutional Review Board (IRB) of D university. The informed consent was obtained from the subjects before data collection. Confidentiality of data collected was ensured.

Source of Funding: The paper was support by the Daewon University College Research Grant of 2020.

Conflict of Interest: Nothing specific-can use the study findings with proper citation of authors name.

\section{References}

1. KimJH.A legal study on the extended application of "Emergency medical fund" to 119 fire service. Unpublished Master's thesis. Dankook University ofCheonan in Korea, 2015.

2. Jo. YH, ShinSD, SuhGJ, Kim JS. The effect of hospital-based emergency medical technician training on the prehospital measurement of vital signs. The Korean Society of EmergencyMedicine 2007;18(4): 267-276.

3. Ministry ofPublic Safety and Security.2021 National Fire Agency 119 Statistical Yearbook.2021. Available online: https://www.nfa.go.kr/nfa/ releaseinformation/statisticalinformation/ main/?boardId=bbs_0000000000000019\& mode $=$ view\&cntId=34 (accessed on 1 Jun 2021).
4. Rho SG, Lee J.K, Kim JH. A research on the actual condition of the prehospital emergency care and education in 119 emergency medical services. Journal of the Korea Academia-Industrial Cooperation Society 2012; 13(5): 2117-2124. http://dx.doi.org/10.5762/KAIS.2012.13.5.2117

5. Hwang SH. Accuracy of blood pressure measurements by 119 Emergency Medical Technician. Unpublished Master's thesis. Korea National University of Chung-Ju in Korea, 2017.

6. YooIS, LeeKH, Hur T, Yoo YH.A study on the revision of ambulance standards and regulations related to emergency patient transportation. Chungnam National University Ministry of Health and Welfare Research Report. Chungnam : NCMIK, 2011.Available online: https://viewer. nanet.go.kr/view/sd;streamdocsId=72059217150 688890; currentPage $=1$; certification $I d=$ de12ea8e$3 e 38-4 d 52-9022-5710 \mathrm{c} 4 \mathrm{c} 8 \mathrm{f} 54 \mathrm{f} ;$; earchWord $=($ acc essed on 2 May 2021).

7. McAlister FA, Straus SE. Evidence based treatment of hypertension. Measurement of blood pressure: an evidence based review. BMJ 2001;322(7291): 908-911. http://dx.doi. org/10.1136/bmj.322.7291.908

8. Seidlerová J, Tůmová $\mathrm{P}$, Rokyta R, Hromadka M. Factors influencing the accuracy of noninvasive blood pressure measurements in patients admitted for cardiogenic shock. BMC Cardiovascular Disorders2019;19(1): 150. http:// dx.doi.org/10.1186/s12872-019-1129-9

9. BaeNY. Comparison of Direct and Indirect Measurements of Blood Pressure. Journal of Clinical Nursing Research 2001: 5: 195-207.

10. Kim MS, Park MH. Knowledge and practice about blood pressure measurement among nursing students. Journal of Gwangju Health University 2003;28: 411-425.

11. Choi ES. Accuracy of blood pressure measurements 
40 Medico-legal Update, January-March 2022, Vol.22, No. 1

taken using a blood pressure simulator by paramedicstudents. The Korean Journal of Emergency Medical Services 2015; 19(1): 7-17. http://dx.doi.org/10.14408/KJEMS.2015.19.1.007

12. JeonJI. A study on the applicability of sphygmomanometer in emergency situations. Unpublished Master's thesis. Seoul University of Seoul in Korea, 2017.

13. BottenbergMM, BryantGA, HaackSL, NorthA.
M. Assessing pharmacy students' ability to accurately measure blood pressure using a blood pressure simulator arm. American Journal Pharmaceutical Education 2013;77(5): 98. http:// dx.doi.org/10.5688/ajpe77598

14. GordonCJ, Frotjold A, Bloomfield JG. Nursing students blood pressure measurement accuracy during clinical practice. Journal of Nursing Education and Practice 2015;5(5): 46-54. http:// dx.doi.org/10.5430/jnep.v5n5p46 